\title{
Bond Dissociation Energies in Heavy Element Chalcogen and Halogen Small Molecules
}

Monica Vasiliu, ${ }^{1}$ Kirk A. Peterson, ${ }^{2}$ and David A. Dixon ${ }^{1, *}, 1$

${ }^{1}$ Department of Chemistry and Biochemistry, University of Alabama, Tuscaloosa, Alabama

35401, USA

${ }^{2}$ Department of Chemistry, Washington State University, Pullman, Washington, 99164, USA

\section{Complete Manuscript References:}

Reference 4. Rothe, S.; Andreyev, A. N.; Antalic, S.; Borschevsky, A.; Capponi, L.; Cocolios, T. E.; De Witte, H.; Eliav, E.; Fedorov, D. V.; Fedosseev, V. N.; Fink, D.A.; Fritzsche, S.; Ghys, L.; Huyse, M.; Imai, N.; Kaldor, U.; Kudryavtsev, Y.; Köster, U.; Lane, J. F. W.; Lassen, J.; Liberati, V.; Lynch, K. M.; Marsh, B. A.; Nishio, K.; Pauwels, D.; Pershina, V.; Popescu, L.; Procter, T. J.; Radulov D.; Raeder, S.; Rajabali, M. M.; Rapisarda, E.; Rossel, R. E.; Sandhu, K.; Seliverstov, M. D.; Sjödin, A. M.; Van den Bergh, P.; Van Duppen, P.; Venhart, M.; Wakabayashi, Y.; Wendt, K. D. A. Measurement of the first ionization potential of astatine by laser ionization spectroscopy. Nature Comm. 2013, 4, 1835-1 - 1835-6.

Reference 5. Leimbach, D.; Karls, J.; Guo, Y.; Ahmed, R.; Ballof, J.; Bengtsson, L.; Pamies, F. B.; Borschevsky, A.; Chrysalidis, K.; Eliav, E.; Fedorov, D.; Fedosseev, V.; Forstner, O.; Galland, N.; Ruiz, R. F. G.; Granados, C.; Heinke, R.; Johnston, K.; Koszorus, A.; Köste, U.; Kristiansson, M. K.; Liu, Y.; Marsh, B.; Molkanov, P.; Pašteka, L. F.; Ramos, J. P.; Renault, E.; Reponen, M.; Ringvall-Moberg, A.; Rossel, R. E.; Studer, D.; Vernon, D.; Warbinek, J.; Welander, J.; Wendt, K.; Wilkins, S.; Hanstorp, D.; Rothe, S. The electron affinity of astatine Nature Comm. 2020, 11, 3824-1 - 3824-9.

Reference 23. Frisch, M. J.; Trucks, G. W.; Schlegel, H. B.; Scuseria, G. E.; Robb, M. A.; Cheeseman, J. R.; Scalmani, G.; Barone, V.; Petersson, G. A.; Nakatsuji, H.; Li, X.; Caricato, M.; Marenich, A. V.; Bloino, J.; Janesko, B. G.; Gomperts, R.; Mennucci, B.; Hratchian, H. P.; Ortiz, J. V.; Izmaylov, A. F.; Sonnenberg, J. L.; Williams-Young, D.; Ding, F.; Lipparini, F.; Egidi, F.; Goings, J.; Peng, B.; Petrone, A.; Henderson, T.; Ranasinghe, D.; Zakrzewski, V. G.; Gao, J.; Rega, N.; Zheng, G.; Liang, W.; Hada, M.; Ehara, M.; Toyota, K.; Fukuda, R.; Hasegawa, J.; Ishida, M.; Nakajima, T.; Honda, Y.; Kitao, O.; Nakai, H.; Vreven, T.; Throssell, K.; Montgomery, J. A., Jr.; Peralta, J. E.; Ogliaro, F.; Bearpark, M. J.; Heyd, J. J.; Brothers, E. N.; Kudin, K. N.; Staroverov, V. N.; Keith, T. A.; Kobayashi, R.; Normand, J.; Raghavachari, K.; Rendell, A. P.; Burant, J. C.; Iyengar, S. S.; Tomasi, J.; Cossi, M.; Millam, J. M.; Klene, M.; Adamo, C.; Cammi, R.; Ochterski, J. W.; Martin, R. L.; Morokuma, K.; Farkas, O.; Foresman, J. B.; Fox, D. J. Gaussian 16, Revision A.03, Gaussian, Inc., Wallingford CT, 2016.

\footnotetext{
${ }^{1}$ Email: dadixon@ua.edu
} 
Reference 47. DIRAC, A Relativistic Ab Initio Electronic Structure Program, Release DIRAC19 (2019), written by Gomes, A. S. P.; Saue, T.; Visscher, L.; Jensen, H. J. Aa.; Bast, R. with contributions from Aucar, I. A.; Bakken, V.; Dyall, K. G.; Dubillard, S.; Ekström, U.; Eliav, E.; Enevoldsen, T.; Faßhauer, E.; Fleig, T.; Fossgaard, O.; Halbert, L.; Hedegård, E. D.; Helgaker, T.; Helmich-Paris, B.; Henriksson, J.; Iliaš, M.; Jacob, Ch. R.; Knecht, S.; Komorovský, S.; Kullie, O.; Lærdahl, J. K.; Larsen, C. V.; Lee, Y. S.; Nataraj, H. S.; Nayak, M. K.; Norman, P.; Olejniczak, G.; Olsen, J.; Olsen, J. M. H.; Park, Y. C.; Pedersen, J. K.; Pernpointner, M.; Di Remigio, R.; Ruud, K.; Sałek, P.; Schimmelpfennig, B.; Senjean, B.; Shee, A.; Sikkema, J.; Thorvaldsen, A. J.; Thyssen, J.; van Stralen, J.; Vidal, M. L.; Villaume, S.; Visser, O.; Winther, T.; Yamamoto S. (available at http://dx.doi.org/10.5281/zenodo.3572669, see also http://www.diracprogram.org).

Reference 62. Werner H.-J.; Knowles, P. J.; Knizia, G.; Manby, F. R.; Schütz, M.; Celani, P.; Györffy, W.; Kats, T.; Korona, T.; Lindh, R.; Mitrushenkov, A.; Rauhut, G.; Shamasundar K. R.; Adler, T. B.; Amos, R. D.; Bennie, S. J.; Bernhardsson, A.; Berning, A.; Cooper, D. L.; Deegan, M. J. O.; Dobbyn, A. J.; Eckert, F.; Goll, E.; Hampel, C.; Hesselmann, A.; Hetzer, G.; Hrenar, T.; Jansen, G.; Köppl, C.; Lee, S. J. R.; Liu, Y.; Lloyd, A. W.; Ma, Q.; Mata, R. A.; May, A. J.; McNicholas, S. J.; Meyer, Miller T. F., III; W.; Mura, M. E.; Nicklass, A.; O'Neill D. P.; Palmieri, P.; Peng, D.; Pflüger, K.; Pitzer, R.; Reiher, M.; Shiozaki, T.; Stoll, H.; Stone, A. J.; Tarroni, R.; Thorsteinsson, T.; Wang, M.; Welborn M. version 2019.2, A Package of $A b$ Initio Programs (https://www.molpro.net) [Accessed September 1, 2020].

Reference 63. Werner, H.-J.; Knowles, P. J.; Manby, F. R.; Black, J. A.; Doll, K.; Heßelmann, A.; Kats, D.; Köhn, A.; Korona, T.; Kreplin, D. A.; Ma, Q.; Miller, T. F.; Mitrushchenkov, A.; Peterson, K. A.; Polyak, I.; Rauhut, G.; Sibaev M. The Molpro Quantum Chemistry Package. J. Chem. Phys. 2020, 152, 144107-1 - 144107-24. 
Table S1. Anharmonicity Constants $\left(\omega_{\mathrm{e}} x_{\mathrm{e}}\right)$ values for the diatomics.

\begin{tabular}{|l|c|l|l|l|l|}
\hline Molecule & State & $\begin{array}{l}\omega_{\mathrm{e}} x_{\mathrm{e}}\left(\mathrm{cm}^{-1}\right) \\
\text { CCSD }(\mathrm{T})^{\mathrm{a}}\end{array}$ & $\Delta \omega_{e} x_{e}\left(\mathrm{~cm}^{-1}\right)^{\mathrm{b}}$ & $\begin{array}{l}\omega_{\mathrm{e}} x_{\mathrm{e}}\left(\mathrm{cm}^{-1}\right) \\
\text { Final }^{\mathrm{c}}\end{array}$ & $\begin{array}{l}\omega_{\mathrm{e}} x_{\mathrm{e}}\left(\mathrm{cm}^{-1}\right) \\
\text { Expt. }\end{array}$ \\
\hline $\mathrm{HSe}$ & ${ }^{2} \Pi_{3 / 2}$ & 42.23 & & 42.2 & \\
\hline $\mathrm{HSe}$ & ${ }^{1} \Sigma_{0}^{+}$ & 45.11 & & 45.1 & \\
\hline $\mathrm{HTe}$ & ${ }^{2} \Pi_{3 / 2}$ & 36.38 & 1.0 & 37.4 & \\
\hline $\mathrm{HTe}^{-}$ & ${ }^{1} \Sigma_{0}^{+}$ & 38.73 & 0.8 & 39.5 & \\
\hline $\mathrm{HPo}$ & ${ }^{2} \Pi_{3 / 2}$ & 33.73 & 2.5 & 36.2 & \\
\hline $\mathrm{HPo}^{-}$ & ${ }^{1} \Sigma_{0}^{+}$ & 35.04 & 4.8 & 39.8 & \\
\hline $\mathrm{Se}_{2}$ & ${ }^{3} \Sigma_{0 g}^{-}$ & 0.89 & & 0.9 & 0.9636 \\
\hline $\mathrm{Te}_{2}$ & ${ }^{3} \Sigma_{0 \mathrm{~g}}^{-}$ & 0.45 & 0.1 & 0.6 & 0.5148 \\
\hline $\mathrm{Po}_{2}$ & ${ }^{3} \Sigma_{0 \mathrm{~g}}^{-}$ & 0.26 & 0.1 & 0.4 & \\
\hline $\mathrm{HBr}$ & ${ }^{1} \Sigma_{0}^{+}$ & 45.07 & & 45.1 & 45.217 \\
\hline $\mathrm{HI}$ & ${ }^{1} \Sigma_{0}^{+}$ & 38.62 & 0.7 & 39.3 & 39.643 \\
\hline $\mathrm{HAt}$ & ${ }^{1} \Sigma_{0}^{+}$ & 35.35 & 3.9 & 39.2 & \\
\hline $\mathrm{Br}_{2}$ & ${ }^{1} \Sigma_{0 \mathrm{~g}}^{+}$ & 1.01 & & 1.0 & 1.0774 \\
\hline $\mathrm{I}_{2}$ & ${ }^{1} \Sigma_{0 \mathrm{~g}}^{+}$ & 0.51 & 0.1 & 0.6 & 0.614 \\
\hline $\mathrm{At}_{2}$ & ${ }^{1} \Sigma_{0 \mathrm{~g}}^{+}$ & 0.28 & 0.2 & 0.5 & \\
\hline
\end{tabular}

${ }^{\mathrm{a}} \mathrm{CCSD}(\mathrm{T}) / \mathrm{awQ}-\mathrm{DK}$ : aug-cc-pwCVQZ-DK3 for Po, At, I, Te; aug-cc-pwCVQZ-DK for Se, Br; aug-cc-pVQZ for H. ${ }^{b}$ Effects of SO+Gaunt on the bond lengths. ${ }^{c}$ Effects of SO+Gaunt added to the $\operatorname{CCSD}(\mathrm{T}) / \mathrm{awQ}-\mathrm{DK}$ bond lengths.

Table S2. Experimental and Calculated (CCSD(T)/awQ-DK) Harmonic Frequencies and Anharmonicity Constants for Triatomics.

\begin{tabular}{|l|l|l|l|l|}
\hline Molecule & $\omega_{\mathrm{e}}\left(\mathrm{cm}^{-1}\right)$ & $v_{\text {Expt. }}{ }^{\text {}}$ & $\omega_{\mathrm{e}} \mathrm{X}_{\mathrm{e}}\left(\mathrm{cm}^{-1}\right)$ & ratio \\
\hline \multirow{3}{*}{$\mathrm{H}_{2} \mathrm{Se}$} & 2437.9 & 2345 & 93 & 0.038 \\
& 1056.6 & 1034 & 23 & 0.022 \\
& 2456.1 & 2358 & 98 & 0.040 \\
\hline \multirow{3}{*}{$\mathrm{H}_{2} \mathrm{Te}$} & 2144.0 & 2065.2709 & 78.7 & 0.037 \\
& 871.4 & 860.6563 & 10.7 & 0.012 \\
& 2157.4 & 2072.1101 & 85.3 & 0.040 \\
\hline \multirow{3}{*}{$\mathrm{H}_{2} \mathrm{Po}$} & 1566.5 & 1504 est & 63 est & 0.038 \\
& 492.2 & 483 est & 9 est & 0.017 \\
& 1689.3 & 1617 est & 72 est & 0.04 \\
\hline
\end{tabular}

${ }^{\mathrm{b}}$ See text References 65,74 , and 75 . 
Table S3. Calculated Chalcogen BDEs in $\mathrm{kcal} / \mathrm{mol}$ for a CBS limit extrapolation of Q and 5. ${ }^{\mathrm{a}}$

\begin{tabular}{|l|c|c|}
\hline $\mathrm{BDE}$ & $\Delta \mathrm{E}(\mathrm{aw} 5)$ & $\Delta \mathrm{E}_{\mathrm{CBS}}(\mathrm{Q}, 5)$ \\
\hline $\mathrm{H}_{2} \mathrm{Se} \rightarrow \mathrm{HSe}+\mathrm{H}$ & 85.0 & 85.3 \\
\hline $\mathrm{HSe} \rightarrow \mathrm{Se}+\mathrm{H}$ & 77.8 & 78.1 \\
\hline $\mathrm{Se}_{2} \rightarrow 2 \mathrm{Se}$ & 52.1 & 83.3 \\
\hline $\mathrm{H}_{2} \mathrm{Se} \rightarrow \mathrm{Se}+2 \mathrm{H}$ & 162.8 & 163.4 \\
\hline $\mathrm{HBr} \rightarrow \mathrm{Br}+\mathrm{H}$ & 93.3 & 93.6 \\
\hline $\mathrm{Br}_{2} \rightarrow 2 \mathrm{Br}$ & 51.6 & 52.6 \\
\hline
\end{tabular}

${ }^{\mathrm{a}} \mathrm{CCSD}(\mathrm{T}) /$ aug-cc-pwCVnZ-DK for $\mathrm{n}=\mathrm{Q}$ and 5 using Eq 2

Table S4. Acidities in $\mathrm{kcal} / \mathrm{mol}$ BDEs in $\mathrm{kcal} / \mathrm{mol}$ for a CBS limit extrapolation of Q and 5. ${ }^{\mathrm{a}}$

\begin{tabular}{|l|l|l|}
\hline & $\Delta \mathrm{E}($ aw5-DK) & $\Delta \mathrm{E}_{\mathrm{CBS}}(\mathrm{Q}, 5-\mathrm{DK})$ \\
\hline $\mathrm{H}_{2} \mathrm{Se} \rightarrow \mathrm{HSe}^{-}+\mathrm{H}^{+}$ & 345.9 & 345.7 \\
\hline $\mathrm{HBr} \rightarrow \mathrm{Br}^{-}+\mathrm{H}^{+}$ & 326.8 & 326.3 \\
\hline
\end{tabular}

${ }^{\mathrm{a}} \mathrm{CCSD}(\mathrm{T}) /$ aug-cc-pwCVnZ-DK for $\mathrm{n}=\mathrm{Q}$ and 5 using Eq 2

Table S5. BDEs in $\mathrm{kcal} / \mathrm{mol}$ when effective core potentials basis sets are used and core-valence corrections in $\mathrm{kcal} / \mathrm{mol}$.

\begin{tabular}{|l|l|l|l|l|}
\hline $\mathrm{BDE}$ & $\Delta \mathrm{E}(\mathrm{awQ}-\mathrm{PP})$ & $\Delta \mathrm{E}(\mathrm{aw} 5-\mathrm{PP})$ & $\Delta \mathrm{E}_{\text {cBS }}(\mathrm{Q}, 5-\mathrm{PP})$ & $\Delta$ EawQ(DK-PP) \\
\hline $\mathrm{HBr} \rightarrow \mathrm{Br}+\mathrm{H}$ & 93.0 & 93.2 & 93.6 & 0.07 \\
\hline $\mathrm{HI} \rightarrow \mathrm{I}+\mathrm{H}$ & 80.1 & 80.2 & 80.4 & 0.07 \\
\hline $\mathrm{HAt} \rightarrow \mathrm{At}+\mathrm{H}$ & 73.2 & 73.3 & 73.4 & -0.12 \\
\hline $\mathrm{Br}_{2} \rightarrow \mathrm{Br}$ & 50.7 & 51.6 & 52.5 & 0.05 \\
\hline $\mathrm{I}_{2} \rightarrow 2 \mathrm{I}$ & 46.5 & 47.5 & 48.6 & 0.10 \\
\hline $\mathrm{At}_{2} \rightarrow 2 \mathrm{At}$ & 42.8 & 43.7 & 44.7 & -0.18 \\
\hline $\mathrm{H}_{2} \mathrm{Se} \rightarrow \mathrm{HSe}+\mathrm{H}$ & 84.9 & 85.1 & 85.3 & -0.07 \\
\hline $\mathrm{HSe} \rightarrow \mathrm{Se}+\mathrm{H}$ & 77.6 & 77.8 & 78.1 & -0.06 \\
\hline $\mathrm{H}_{2} \mathrm{Se} \rightarrow \mathrm{Se}+2 \mathrm{H}$ & 162.4 & 162.9 & 163.5 & -0.14 \\
\hline $\mathrm{H}_{2} \mathrm{Te} \rightarrow \mathrm{HTe}+\mathrm{H}$ & 74.7 & 74.8 & 74.9 & -0.27 \\
\hline $\mathrm{HTe} \rightarrow \mathrm{Te}+\mathrm{H}$ & 68.9 & 69.0 & 69.2 & -0.22 \\
\hline $\mathrm{H}_{2} \mathrm{Te} \rightarrow \mathrm{Te}+2 \mathrm{H}$ & 143.6 & 143.9 & 144.1 & -0.49 \\
\hline $\mathrm{H}_{2} \mathrm{Po} \rightarrow \mathrm{HPo}+\mathrm{H}$ & 68.1 & 68.1 & 68.2 & -0.07 \\
\hline $\mathrm{HPo} \rightarrow \mathrm{Po}+\mathrm{H}$ & 63.2 & 63.3 & 63.5 & -0.07 \\
\hline $\mathrm{H}_{2} \mathrm{Po} \rightarrow \mathrm{Po}+2 \mathrm{H}$ & 131.2 & 131.5 & 131.7 & -0.15 \\
\hline $\mathrm{Se}_{2} \rightarrow 2 \mathrm{Se}$ & 81.1 & 82.3 & 83.6 & -0.21 \\
\hline $\mathrm{Po}_{2} \rightarrow 2 \mathrm{Po}$ & 60.0 & 61.2 & 62.3 & -0.25 \\
\hline $\mathrm{Te}_{2} \rightarrow 2 \mathrm{Te}$ & 68.0 & 69.2 & 70.5 & -0.47 \\
\hline
\end{tabular}


Table S6. Acidities when effective core potentials basis sets are used and core-valence corrections in $\mathrm{kcal} / \mathrm{mol}$.

\begin{tabular}{|l|l|l|l|l|}
\hline $\mathrm{Reaction}$ & $\Delta \mathrm{E}(\mathrm{awQ}-\mathrm{PP})$ & $\Delta \mathrm{E}(\mathrm{aw} 5-\mathrm{PP})$ & $\Delta \mathrm{E}_{\mathrm{CBS}}(\mathrm{Q}, 5-\mathrm{PP})$ & $\Delta$ EawQ(DK-PP) \\
\hline $\mathrm{H}_{2} \mathrm{Se} \rightarrow \mathrm{HSe}^{-}+\mathrm{H}^{+}$ & 346.3 & 346.0 & 345.8 & -0.13 \\
\hline $\mathrm{H}_{2} \mathrm{Te} \rightarrow \mathrm{HTe}^{-}+\mathrm{H}^{+}$ & 335.8 & 335.4 & 334.9 & -0.31 \\
\hline $\mathrm{H}_{2} \mathrm{Po} \rightarrow \mathrm{HPo}^{-}+\mathrm{H}^{+}$ & 331.8 & 331.4 & 330.9 & -0.15 \\
\hline $\mathrm{HBr} \rightarrow \mathrm{Br}^{-}+\mathrm{H}^{+}$ & 327.2 & 326.8 & 326.3 & -0.01 \\
\hline $\mathrm{HI} \rightarrow \mathrm{I}^{-}+\mathrm{H}^{+}$ & 318.1 & 317.5 & 316.8 & -0.03 \\
\hline $\mathrm{HAt} \rightarrow \mathrm{At}^{-}+\mathrm{H}^{+}$ & 315.3 & 314.6 & 313.8 & -0.17 \\
\hline
\end{tabular}

Table S7. Atomic entropies (S) in cal/mol K (at $298 \mathrm{~K}$ ).

\begin{tabular}{|l|c|}
\hline Atom & $\mathrm{S}$ \\
\hline $\mathrm{Br}$ & 41.80 \\
\hline $\mathrm{I}$ & 43.18 \\
\hline $\mathrm{At}$ & 44.68 \\
\hline $\mathrm{Se}$ & 42.21 \\
\hline $\mathrm{Te}$ & 43.64 \\
\hline $\mathrm{Po}$ & 45.12 \\
\hline
\end{tabular}


Table S8. Calculated entropies (S) in cal/mol K (at $298 \mathrm{~K}$ ).

\begin{tabular}{|l|c|}
\hline Molecule & $\mathrm{S}(\mathrm{cal} / \mathrm{mol} \mathrm{K})$ \\
\hline $\mathrm{H}_{2} \mathrm{Se}$ & 52.34 \\
\hline $\mathrm{HSe}$ & 47.59 \\
\hline $\mathrm{HSe}^{-}$ & 47.60 \\
\hline $\mathrm{H}_{2} \mathrm{Te}$ & 54.63 \\
\hline $\mathrm{HTe}$ & 49.52 \\
\hline $\mathrm{HTe}^{-}$ & 49.54 \\
\hline $\mathrm{H}_{2} \mathrm{Po}$ & 56.95 \\
\hline $\mathrm{HPo}^{-}$ & 51.19 \\
\hline $\mathrm{HPo}^{-}$ & 51.22 \\
\hline $\mathrm{Se}_{2}$ & 58.60 \\
\hline $\mathrm{Te}_{2}$ & 61.94 \\
\hline $\mathrm{Po}_{2}$ & 65.44 \\
\hline $\mathrm{HBr}$ & 47.41 \\
\hline $\mathrm{HI}$ & 49.34 \\
\hline $\mathrm{HAt}$ & 51.13 \\
\hline $\mathrm{Br}_{2}$ & 58.56 \\
\hline $\mathrm{I}_{2}$ & 62.25 \\
\hline $\mathrm{At}_{2}$ & 66.40 \\
\hline
\end{tabular}


Table S9. Electronic energies in Hartrees at different level of theory. ${ }^{\mathrm{a}}$

\begin{tabular}{|l|l|l|l|l|l|}
\hline Molecule & awT-DK & awQ-DK & aw5-DK & CBS(T,Q-DK) & CBS(Q,5-DK) \\
\hline $\mathrm{H}_{2} \mathrm{Se}$ & -2430.086788 & -2430.257428 & -2430.328382 & -2430.355919 & -2430.402825 \\
\hline $\mathrm{HSe}$ & -2429.452788 & -2429.622263 & -2429.692853 & -2429.720080 & -2429.766916 \\
\hline $\mathrm{HSe}^{-}$ & -2429.533710 & -2429.705770 & -2429.777105 & -2429.805079 & -2429.851948 \\
\hline $\mathrm{H}_{2} \mathrm{Te}$ & -6793.216111 & -6793.364057 & & -6793.449449 & \\
\hline $\mathrm{HTe}$ & -6792.598397 & -6792.745382 & & -6792.830218 & \\
\hline $\mathrm{HTe}{ }^{-}$ & -6792.679635 & -6792.829419 & & -6792.915871 & \\
\hline $\mathrm{H}_{2} \mathrm{Po}$ & -22202.634128 & -22202.888369 & & -22203.035112 & \\
\hline $\mathrm{HPo}$ & -22202.026535 & -22202.280027 & & -22202.426338 & \\
\hline $\mathrm{HPo}^{-}$ & -22202.103541 & -22202.359769 & & -22202.507658 & \\
\hline $\mathrm{Se}_{2}$ & -4857.785788 & -4858.126511 & -4858.268625 & -4858.323169 & -4858.417729 \\
\hline $\mathrm{Te}_{2}$ & -13584.083458 & -13584.379643 & & -13584.550594 & \\
\hline $\mathrm{Po}_{2}$ & -44402.944963 & -44403.454186 & & -44403.748099 & \\
\hline $\mathrm{HBr}$ & -2605.845549 & -2606.019325 & -2606.092687 & -2606.119625 & -2606.169656 \\
\hline $\mathrm{HI}$ & -7114.508508 & -7114.661740 & & -7114.750182 & \\
\hline $\mathrm{HAt}$ & -22878.627207 & -22878.896940 & & -22879.052624 & \\
\hline $\mathrm{Br}_{2}$ & -5210.474350 & -5210.823000 & -5210.970224 & -5211.024234 & -5211.124688 \\
\hline $\mathrm{I}_{2}$ & -14227.834091 & -14228.142379 & & -14228.320316 & \\
\hline $\mathrm{At}_{2}$ & -45756.087513 & -45756.628988 & & -45756.941516 & \\
\hline
\end{tabular}

${ }^{\text {a }}$ aug-cc-pwCVnZ-DK3 for Po, At, I, Te, aug-cc-pwCVnZ-DK for Se, Br, and aug-cc-pVnZ for $\mathrm{H}$ for $\mathrm{n}=\mathrm{T}, \mathrm{Q}$, and 5 
Table S10. Electronic energies in Hartrees at different level of theory. ${ }^{\mathrm{a}}$

\begin{tabular}{|l|l|l|l|}
\hline Molecule & awQ-PP & aw5-PP & CBS(Q,5-PP) \\
\hline $\mathrm{H}_{2} \mathrm{Se}$ & -374.1624419 & -374.234224 & -374.309536 \\
\hline $\mathrm{HSe}$ & -373.5271632 & -373.5986018 & -373.673554 \\
\hline $\mathrm{HSe}^{-}$ & -373.6105775 & -373.6827628 & -373.758498 \\
\hline $\mathrm{H}_{2} \mathrm{Te}$ & -269.240299 & -269.316228 & -269.395890 \\
\hline $\mathrm{HTe}$ & -268.621188 & -268.696976 & -268.776492 \\
\hline $\mathrm{HTe}^{-}$ & -268.705175 & -268.781798 & -268.862189 \\
\hline $\mathrm{H}_{2} \mathrm{Po}$ & -238.845938 & -238.914395 & -238.986220 \\
\hline $\mathrm{HPo}$ & -238.237477 & -238.305792 & -238.377466 \\
\hline $\mathrm{HPo}^{-}$ & -238.317102 & -238.386303 & -238.458908 \\
\hline $\mathrm{Se}_{2}$ & -745.9364379 & -746.0803106 & -746.231259 \\
\hline $\mathrm{Te}_{2}$ & -536.131293 & -536.284252 & -536.444733 \\
\hline $\mathrm{Po}_{2}$ & -475.369252 & -475.507190 & -475.651911 \\
\hline $\mathrm{HBr}^{\mathrm{HI}}$ & -417.2263154 & -417.3001432 & -417.377602 \\
\hline $\mathrm{HAt}$ & -296.357641 & -296.435857 & -296.517919 \\
\hline $\mathrm{Br}_{2}$ & -262.944070 & -263.015985 & -263.091438 \\
\hline $\mathrm{I}_{2}$ & -833.2371216 & -833.385308 & -833.540782 \\
\hline $\mathrm{At}_{2}$ & -591.534242 & -591.691781 & -591.857067 \\
\hline
\end{tabular}

a aug-cc-pwCVnZ-PP for Po, At, I, Te, Se, Br, and aug-cc-pVnZ for $\mathrm{H}$ for $\mathrm{n}=\mathrm{Q}$ and 5 using Eq. 2. 